Nonlinear Processes in Geophysics (2005) 12: 299-310

SRef-ID: $1607-7946 / \mathrm{npg} / 2005-12-299$

European Geosciences Union

(c) 2005 Author(s). This work is licensed

under a Creative Commons License.

\title{
Parallel and perpendicular cascades in solar wind turbulence
}

\author{
S. Oughton ${ }^{1}$ and W. H. Matthaeus ${ }^{2}$ \\ ${ }^{1}$ Department of Mathematics, University of Waikato, Hamilton, New Zealand \\ ${ }^{2}$ Bartol Research Institute, University of Delaware, Newark, DE 19716, USA
}

Received: 27 September 2004 - Revised: 10 February 2005 - Accepted: 12 February 2005 - Published: 17 February 2005

Part of Special Issue "Nonlinear processes in solar-terrestrial physics and dynamics of Earth-Ocean-System"

\begin{abstract}
MHD-scale fluctuations in the velocity, magnetic, and density fields of the solar wind are routinely observed. The evolution of these fluctuations, as they are transported radially outwards by the solar wind, is believed to involve both wave and turbulence processes. The presence of an average magnetic field has important implications for the anisotropy of the fluctuations and the nature of the turbulent wavenumber cascades in the directions parallel and perpendicular to this field. In particular, if the ratio of the rms magnetic fluctuation strength to the mean field is small, then the parallel wavenumber cascade is expected to be weak and there are difficulties in obtaining a cascade in frequency. The latter has been invoked in order to explain the heating of solar wind fluctuations (above adiabatic levels) via energy transfer to scales where ion-cyclotron damping can occur.

Following a brief review of classical hydrodynamic and magnetohydrodynamic (MHD) cascade theories, we discuss the distinct nature of parallel and perpendicular cascades and their roles in the evolution of solar wind fluctuations.
\end{abstract}

\section{Introduction}

The solar wind exhibits fluctuations in the magnetic field, plasma velocity, and density over a broad range of length and time scales, as was suggested by Parker (1958) in connection with his original model for the (large-scale) solar wind. Many of these fluctuations occur at magnetohydrodynamic (MHD) scales, although dissipative processes almost certainly require account to be taken of plasma-scale or kinetic effects.

The nature of the MHD-scale fluctuations is a question of some interest. Analysis of even the earliest observations provided evidence for the presence of both waves and turbulence (e.g. Coleman, 1968; Belcher and Davis, 1971), and it now seems clear that Alfvén waves and turbulence are pervasive

Correspondence to: S. Oughton

(seano@waikato.ac.nz) features of the interplanetary medium, although their relative importance has yet to be fully determined (e.g. Matthaeus et al., 1995; Goldstein et al., 1995; Tu and Marsch, 1995; Veltri and Malara, 1997; Velli et al., 2003).

Both the wave and turbulence dynamics are influenced by the preferred directions present in the interplanetary medium. These include the radial expansion direction and the mean magnetic field. In particular, there is evidence for spectral and/or variance anisotropy with respect to one or both of these preferred directions (e.g. Belcher and Davis, 1971; Klein et al., 1991; Matthaeus et al., 1990, 1996; Bieber et al., 1996; Carbone et al., 1995; Horbury et al., 1995).

As is well-known, the wavenumber energy spectrum for a turbulent fluid can be characterized in terms of three different wavenumber ranges, namely the energy-containing, inertial, and dissipation ranges (e.g. Lesieur, 1990; Frisch, 1995; Biskamp, 2003). Figure 1 shows a schematic solar wind spectrum. In the classical view of turbulence, energy is transferred from the energy-containing scales to the dissipation range scales not directly, but rather via passage through the inertial range "pipeline". In other words, the energy cascades from wavenumber to (somewhat) larger wavenumber until it reaches scales where the direct effects of dissipation are important. The inertial range dynamics is self-similar which yields a powerlaw energy spectrum there, for large enough Reynolds numbers. The question then arises, what can be determined about the nature of solar wind fluctuations from observable quantities like the energy spectrum?

Interestingly, even though the solar wind is a supersonic, fully ionized, anisotropic, collisionless plasma, the inertial range slope is often observed to be $\approx-5 / 3$, which is the value Kolmogorov theory predicts for an incompressible isotropic Navier-Stokes fluid. As yet there is no clear consensus as to why this should be, although various explanations have been suggested. Here, we review some of these suggestions, with a focus on the distinction between the turbulent cascades occuring along and perpendicular to the mean magnetic field. 


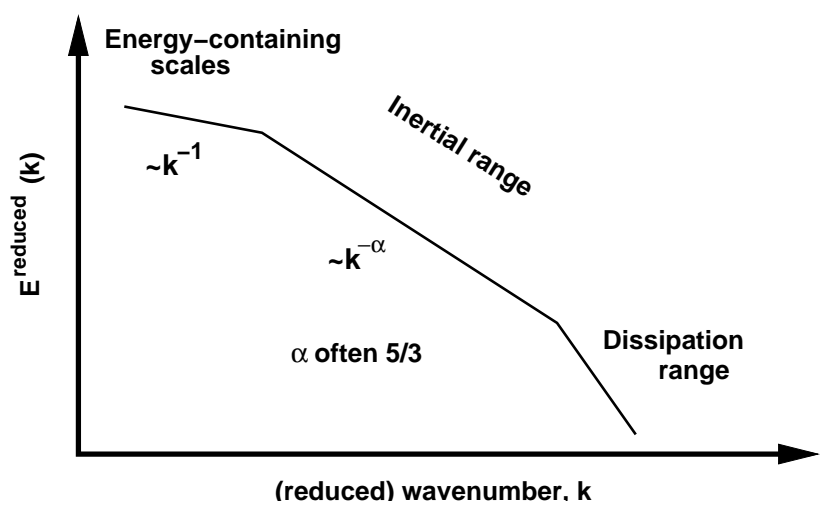

Fig. 1. Schematic energy spectrum for the fluctuation energy of the solar wind. The energy-containing, inertial, and dissipation ranges are indicated. Note the powerlaw nature of the spectrum in the inertial range.

The paper is structured as follows. In the next section we review cascade theory for MHD turbulence. Section 3 considers observational evidence for the existence of two distinct types of fluctuations in the solar wind. In Sect. 4 a simple model is employed to show that slopes of $5 / 3$ can be common even when there is a non-5/3 component. The paper closes with a short summary.

The basic notation employed is standard, with $\boldsymbol{v}$ and $\boldsymbol{b}$ respectively the fluctuating velocity and magnetic fields, $\rho$ the mass density, and $\boldsymbol{k}$ the Fourier wavevector conjugate to the spatial position vector $\boldsymbol{x}$. Magnetic fields are assumed to be measured in Alfvén speed units, obtained starting from laboratory units by letting $\boldsymbol{b} \rightarrow \boldsymbol{b} / \sqrt{4 \pi \rho}$.

\section{MHD cascade theory}

In some ways the physics of (Navier-Stokes) turbulence is more about lengthscales then timescales, since the fundamental action of the nonlinear terms is to transfer excitation between lengthscales. Thinking in Fourier space, this could be rephrased as the primacy of the wavevector spectrum over the frequency spectrum. Of course, each lengthscale (or wavevector) has one or more timescales associated with it, so that there is also an inherent transfer of energy between timescales. In particular, the rates of energy transfer are of importance, helping to determine cascade properties and the shape of the energy spectrum. Indeed, as we review below, Kolmogorov theory can be reformulated in terms of such timescales, showing that it is the triple correlation timescale, $\tau_{3}(\boldsymbol{k})$, that determines the slope of the energy spectrum in the inertial range. In MHD turbulence, as contrasted with Navier-Stokes, the situation is further complicated by the presence of Alfvén waves and their associated timescales.

In the subsections below we first review Kolmogorov theory for hydrodynamics and the Iroshnikov-Kraichnan (IK) extension of it to incompressible MHD, and then summa- rize work addressing the primary shortcoming of the IK approach, namely the assumption of isotropy. Subsections on compressible anisotropy results and forcing/inhomogeneity related timescales round out the section.

\subsection{Kolmogorov (hydrodynamic) phenomenology}

Suppose that an incompressible Navier-Stokes fluid ${ }^{1}$ is forced isotropically at some set of large length and time scales so that a statistically steady turbulent flow is set up. The Kolmogorov (1941) $k^{-5 / 3}$ form for the inertial range energy spectrum can be obtained via dimensional analysis and the assumption that there is a range of lengthscales for which (i) direct viscous damping is negligible, ${ }^{2}$ (ii) the energy flux at wavenumber $k$, denoted $\epsilon(k)$, depends only on local quantities, namely $k$ and the spectrum $E(k)$, and (iii) the flux of energy through this range is in fact a constant. Also implicit is the assumption that the energy is distributed isotropically. Given this isotropic situation, it is convenient to work with the omni-directional (or angle-integrated) spectrum. This is defined by

$E(k)=\int E^{3 \mathrm{D}}(\boldsymbol{k}) k^{2} \mathrm{~d} \Omega$,

where $E^{3 \mathrm{D}}(\boldsymbol{k})$ is the modal energy spectrum and $\mathrm{d} \Omega$ the differential solid angle. The total turbulence energy is $\int_{0}^{\infty} E(k) \mathrm{d} k$, so that $E(k)$ is readily interpreted as the energy per wavenumber at $k$.

Assuming that $\epsilon(k)=k^{\alpha} E(k)^{\beta}$, for constants $\alpha$ and $\beta$, and employing the above assumptions then yields $E(k)=\epsilon^{2 / 3} k^{-5 / 3}$, to within an undetermined $O(1)$ constant usually called the Kolmogorov constant. For fuller discussion of hydrodynamic turbulence see, for example, Batchelor (1970); Lesieur (1990); Frisch (1995).

The Kolmogorov form can also be obtained somewhat more physically, by reformulating the approach to take explicit account of the relevant ( $k$-dependent) timescales. In order to do so we first define these timescales.

There are (at least) three conceptually distinct timescales associated with each wave vector $\boldsymbol{k}$.

- The nonlinear time is $\tau_{\mathrm{NL}}(\boldsymbol{k}) \approx 1 /\left(k u_{\boldsymbol{k}}\right)$, where $u_{\boldsymbol{k}}^{2}$ is approximately the kinetic energy per mass in wavevectors $\sim \boldsymbol{k}$, and represents the timescale associated with nonlinear modification of $u_{\boldsymbol{k}}$.

- The triple correlation timescale $\tau_{3}(\boldsymbol{k})$ characterizes the time separation over which third-order correlations (written symbolically as $\left\langle u u u^{\prime}\right\rangle$ with $u$ and $u^{\prime}$ at different times) tend to zero.

- The spectral transfer timescale $\tau_{\mathrm{s}}(\boldsymbol{k})$ is the time for a significant fraction of the energy in wavevectors $\approx \boldsymbol{k}$ to

\footnotetext{
${ }^{1}$ Meaning gravity, Coriolis force, etc. play no important role.

${ }^{2}$ Thus viscosity is not a relevant parameter in the inertial range.
} 
be transfered to other wavevectors. A common definition is via (Obukhov, 1941)

$$
\frac{\mathrm{d}}{\mathrm{d} t} u_{\boldsymbol{k}}^{2}=-\epsilon(\boldsymbol{k}) \approx-\frac{u_{\boldsymbol{k}}^{2}}{\tau_{\mathrm{s}}} .
$$

Although in general functions of the full wavevector, insisting on isotropy means that the timescales, like the spectra themselves, are then functions of $k=|\boldsymbol{k}|$.

As noted by Kraichnan (1965), the energy flux should be proportional to the triple correlation timescale $\tau_{3}(\boldsymbol{k})$. Assuming isotropy and matching the dimensions of $\epsilon=\tau_{3}(k) k^{\alpha} E(k)^{\beta}$ yields

$\epsilon=\tau_{3}(k) k^{4} E(k)^{2}$,

which might, mnemonically, be called the "2-3-4" rule. In isotropic hydrodynamics the three timescales are in fact equivalent: $\tau_{\mathrm{s}} \approx \tau_{3} \approx \tau_{\mathrm{NL}} \approx 1 /\left(k u_{k}\right) \approx 1 /[k \sqrt{k E(k)}]$, and substitution into Eq. (3) yields $E(k)=\epsilon^{2 / 3} k^{-5 / 3}$, as before. ${ }^{3}$ An advantage of this approach, however, is that it allows for more general situations in which the timescales differ, such as MHD which we discuss next.

\subsection{Iroshnikov-Kraichnan (IK) phenomenology}

Iroshnikov (1964) and Kraichnan (1965) independently suggested that for MHD turbulence with a strong large-scale magnetic field ${ }^{4} \boldsymbol{B}_{0}$, a new timescale becomes important. This is the Alfvén timescale,

$\tau_{\mathrm{A}}(\boldsymbol{k})=\frac{1}{\left|\boldsymbol{k} \cdot \boldsymbol{B}_{0}\right|}=\frac{1}{k\left|B_{0} \cos \theta\right|}$,

and is essentially the period of an Alfvén wave with wavevector $\boldsymbol{k}$. Treating $\cos \theta$ as approximately constant (i.e. neglecting the anisotropy of $\left.\tau_{\mathrm{A}}\right)$, Kraichnan argued that since a strong $B_{0}$ means that $1 /\left(k B_{0}\right)$ is very short, $\tau_{\mathrm{A}}$ should provide the dominant contribution to the triple correlation time in MHD turbulence. Inserting $\tau_{3}(k)=1 /\left(k B_{0}\right)$ in Eq. (3) yields the IK form

$$
E(k)=\sqrt{\epsilon B_{0}} k^{-3 / 2}
$$

Physically, visualizing inertial range MHD turbulence as the interaction of counter-propagating ${ }^{5}$ Alfvén waves, it is clear that their large propagation speed limits the collision time of two such wave-packets to be $\sim \tau_{\mathrm{A}} \ll \tau_{\mathrm{NL}}$. The latter inequality holds if the fluctuation amplitude at scale $\boldsymbol{k}, u_{\boldsymbol{k}}$, is much smaller than the large-scale Alfvén speed $B_{0}$. Let $N(\boldsymbol{k})=\tau_{\mathrm{NL}}(\boldsymbol{k}) / \tau_{\mathrm{A}}(\boldsymbol{k})$ be the number of wave periods in a

\footnotetext{
${ }^{3}$ The approximation $u_{k}^{2} \approx k E(k)$ holds well when $E(k)$ is a powerlaw (e.g. Lesieur, 1990, §6.4.1).

${ }^{4}$ The Iroshnikov and Kraichnan derivations are not identical. For example, Kraichnan considered incompressible MHD while Iroshnikov considered the plasma beta $\approx 1$ compressible situation. Neither author required that a dc field was present.

${ }^{5}$ In incompressible MHD, Alfvén waves propagating in the same direction do not interact, either linearly or nonlinearly.
}

nonlinear time. In order to achieve the equivalent of a hydrodynamic "collision" (i.e. a nonlinear interaction lasting for $\left.\approx \tau_{\mathrm{NL}}\right), N^{2}$ interactions of length $\tau_{\mathrm{A}}$ are required, since the collisions are incoherent. Thus $\tau_{\mathrm{s}}=N^{2} \tau_{\mathrm{A}}=\tau_{\mathrm{NL}}^{2} / \tau_{\mathrm{A}} \gg \tau_{\mathrm{NL}}$, i.e. spectral transfer is slowed down by the presence of Alfvén waves. Substituting this into $\epsilon=u_{k}^{2} / \tau_{\mathrm{s}}(k)=\mathrm{const}$ yields, as before, the IK form $E(k) \sim k^{-3 / 2}$.

Although well-regarded for many years, it is now recognized that there is an important problem with the IK approach, namely the neglect of the intrinsic anisotropy of $\tau_{\mathrm{A}}(\boldsymbol{k})$ with respect to $\boldsymbol{B}_{0}$. There are many wavevectors for which $\boldsymbol{k} \cdot \boldsymbol{B}_{0} \not \approx k B_{0}$; in particular, modes with $\boldsymbol{k} \perp \boldsymbol{B}_{0}$ have $\tau_{\mathrm{A}}(\boldsymbol{k}) \rightarrow \infty$, which is not a short timescale and so will not dominate the contribution to $\tau_{3}$ for those $\boldsymbol{k s}$. (Modes with $\boldsymbol{k} \cdot \boldsymbol{B}_{0}=0$ are called the two-dimensional (2D) modes, while those with $\boldsymbol{k} \cdot \boldsymbol{B}_{0} \approx 0$ are the quasi-2D modes. See Oughton et al. (2004) for discussion of how small $\boldsymbol{k} \cdot \boldsymbol{B}_{0}$ needs to be.)

Thus the cascade in directions perpendicular and parallel to $\boldsymbol{B}_{0}$ is likely to be different, engendering a non-isotropic modal spectrum. Consequently, the relevance of the IK phenomenology to MHD turbulence is probably more limited than was initially thought. In addition, the omni-directional spectrum does not have the clean interpretation pertaining in isotropic cases since excitations at different directions to $\boldsymbol{B}_{0}$ but with the same $|\boldsymbol{k}|$ are lumped together.

Models designed to take some account of this anisotropy have been proposed (Pouquet et al., 1976; Grappin et al., 1982; Matthaeus and Zhou, 1989). The key idea is that the rate of decorrelation of triples is

$\frac{1}{\tau_{3}} \approx \frac{1}{\tau_{\mathrm{NL}}}+\frac{1}{\tau_{\mathrm{A}}}$,

that is, the sum of the decorrelation rates from distinct physical effects. In this case, decorrelation occurs due to the usual advective effects (rate $\sim 1 / \tau_{\mathrm{NL}}$ ) and also via the limited time for which counter-propagating wave-packets are in contact. This simple model provides a bridge between the Kolmogorov spectrum $\left(\tau_{\mathrm{A}} \rightarrow \infty\right)$ and the IK spectrum $\left(B_{0} \rightarrow \infty\right)$ for the over-simplistic approximation $\boldsymbol{k} \cdot \boldsymbol{B}_{0} \approx k B_{0}$.

If instead the full anisotropic $\tau_{\mathrm{A}}(\boldsymbol{k})$ is used in Eq. (6) and one tries to substitute this into a version of Eq. (3) based on $E^{3 \mathrm{D}}(\boldsymbol{k})$ rather than $E(k)$, there is an immediate problem since there are now (at least) two lengthscales present, $k_{\perp}$ and $k_{\|}=k \cos \theta$, which dimensional analysis cannot distinguish between.

Note that there is also another difference between MHD and hydrodynamic turbulence. The above phenomenologies all assume that the normalized cross helicity,

$\sigma_{c}=\frac{2\langle\boldsymbol{v} \cdot \boldsymbol{b}\rangle}{\left\langle\boldsymbol{v}^{2}\right\rangle+\left\langle\boldsymbol{b}^{2}\right\rangle}$,

is small $(\ll 1)$. This is not a necessary physical requirement, however. Extensions to take account of significant cross helicity levels have been considered (e.g. Dobrowolny et al., 1980; Grappin et al., 1982, 1983; Pouquet et al., 1986; Matthaeus et al., 1994; Hossain et al., 1995). 
2.3 Anisotropy of the turbulence spectrum and its consequences

When conditions are such that a large-scale mean magnetic field $\boldsymbol{B}_{0}$ threads the plasma, the dynamics, including turbulence if it occurs, can be expected to respond to this preferred direction, frequently through development of anisotropy. This is seen even in the most basic models of linear MHD waves, where dispersion relations involve anisotropic terms like $\boldsymbol{k} \cdot \boldsymbol{B}_{0} . \quad$ In the simplest anisotropic model of fluctuation symmetry ${ }^{6}$ - the "slab model" - excited wavevectors $\boldsymbol{k}$ lie along $\boldsymbol{B}_{0}$ and the spectrum is one-dimensional. The slab model has been widely employed in cosmic ray scattering theory (Jokipii, 1966) and in interpretations of spacecraft data (Belcher and Davis, 1971), and elsewhere, but it is undoubtedly too simple. For example, for incompressible MHD, the slab model allows no wave-wave couplings, and therefore no possibility of turbulence or a Kolmogorov-like cascade.

The overemphasis on the slab model in solar wind applications probably derives from two unfortunate oversimplifications. First, the "Alfvénic" fluctuations often observed in the solar wind (e.g. Belcher and Davis, 1971) are identified by their high degree of correlation of the fluctuating components of the magnetic and velocity field; in turbulence terms these are high cross helicity states. Such fluctuations resemble wave normal modes of MHD for fluctuations propagating along the locally dc magnetic field $\boldsymbol{B}_{0}$; these might be assumed to obey a dispersion relation $\omega= \pm \boldsymbol{k} \cdot \boldsymbol{B}_{0}$ for the frequency $\omega$, and to have perfectly correlated (or anticorrelated) velocity and magnetic perturbations, depending upon the sign of frequency. Second the fluctuation variances in each of the two directions transverse to $\boldsymbol{B}_{0}$, tend to be larger (by about a factor of five) than the parallel variance (Belcher and Davis, 1971; Klein et al., 1991; Horbury et al., 1995). The so-called "minimum variance direction" argument proceeds to estimate the direction of $\boldsymbol{k}$ as parallel to the direction of minimum variance, i.e. $\boldsymbol{B}_{0}$. Taken together, one concludes that the "turbulence" (which cannot be turbulence at all in the usual sense) has slab symmetry.

However, in strong MHD turbulence the cross helicity enters into the physics as well (e.g. Dobrowolny et al., 1980), and dispersion relations do not provide the time dependence in this case (i.e. there are many frequencies associated with each $\boldsymbol{k}$ ). In addition, the minimum variance argument, by itself, cannot determine the direction of $\boldsymbol{k}$. Consider, for example, a total magnetic field $\boldsymbol{B}=\left(b_{x}, b_{y}, B_{0}\right)$ with fluctuations $b_{x}=\partial a(x, y) / \partial y$ and $b_{y}=-\partial a(x, y) / \partial x$ that are transverse to the mean field. The wavevectors, however, are clearly perpendicular - not parallel - to $\boldsymbol{B}_{0}$. Since such fluctua-

\footnotetext{
${ }^{6}$ The most frequent assumption, that of isotropy, postulates equal excitation in all wavevectors $\boldsymbol{k}$ with the same $|\boldsymbol{k}|$. Classical hydrodynamic turbulence theory (e.g. Batchelor, 1970) is almost entirely based on this assumption, and applications of it to the solar wind have sometimes made a tacit assumption of isotropy (e.g. Coleman, 1968; Tu et al., 1984).
}

tions have wavevectors lying in the plane perpendicular to the mean field, they are often called 2D fluctuations.

Besides being a counterexample to the minimum variance argument, 2D fluctuations form a kind of paradigm for turbulence, in much the same way that slab fluctuations represent the essence of MHD Alfvén wave physics. 2D fluctuations have $\boldsymbol{k} \cdot \boldsymbol{B}_{0}=0$ and are of "zero frequency."7 Therefore the Alfvénic time decorrelation that entered into the discussion in Sect. 2.2 does not occur: the decorrelation of 2D fluctuations occurs without influence of the out-of-plane magnetic field $\boldsymbol{B}_{0}$. Uninhibited by this wave propagation effect, 2D turbulence can be expected to be relatively stronger than other turbulence in which the nonlinear couplings decay in part due to propagation effects. ${ }^{8}$

Although 2D symmetry is itself another highly idealized case, it points towards families of symmetries that may in fact be relevant to MHD with a strong mean field. For example, it is well known in laboratory plasma studies (Zweben et al., 1979; Robinson and Rusbridge, 1971) that the correlation scales along the mean field are much longer than those perpendicular to the mean field. This led to development of socalled "Reduced MHD" models (RMHD) which are "quasi$2 \mathrm{D}$ " in the sense that they have excited wavevectors only in a region of $\boldsymbol{k}$-space near $\boldsymbol{k} \cdot \boldsymbol{B}_{0} \approx 0$. They are also incompressible (or nearly so). Various derivations of RMHD (Kadomtsev and Pogutse, 1974; Strauss, 1976; Montgomery, 1982; Zank and Matthaeus, 1992a), suggest how this kind of lowfrequency quasi-2D dynamics may be the "leading-order" description of nonlinear evolution of MHD in the presence of a strong guide field (cf. Montgomery and Turner, 1981). The main point of the derivations of RMHD is that the strongest nonlinearities - and therefore the expectation of the strongest wavenumber cascade - will occur in regions of $\boldsymbol{k}$-space for which the nonlinear time is less than the Alfvén time, i.e. $\tau_{\mathrm{NL}}(\boldsymbol{k}) \leq \tau_{\mathrm{A}}(\boldsymbol{k})$.

An interesting and recurring topic has been the study of the boundaries of applicability of the RMHD model. Montgomery (1982) noted that strong anisotropy of spectral transfer leads to "freezing out" of parallel spectral transfer, so that $k_{\|}$no longer increases - in this limit there is no parallel cascade at all. Higdon (1984) recognized that quasi-2D turbulence confined within a dynamically determined region in $\boldsymbol{k}$-space would have, in steady-state, a distinctive boundary shape in which the maximum (or, typical) excited $k_{\perp}$ is related to the maximum excited $k_{\|}$. Later this was elaborated upon by Goldreich and Sridhar $(1995,1997)$ who made use of these ideas to note that the marginal condition of $\tau_{\mathrm{NL}}(\boldsymbol{k})=\tau_{\mathrm{A}}(\boldsymbol{k})$ would take on the powerlaw form $k_{\perp} \sim k_{\|}^{3 / 2}$ for steady MHD turbulence with a $k^{-5 / 3}$ inertial range (Higdon also obtained this scaling). They refer to this as a "criti-

\footnotetext{
7 Here frequency means wave frequency, not a general (e.g. Fourier) decomposition of the time dependence.

${ }^{8}$ Propagation effects can enter the physics of the 2D turbulence if the large-scale fluctuating field in the plane is sufficiently strong, but this is distinct from decorrelation associated with the strength of $\boldsymbol{B}_{0}$.
} 

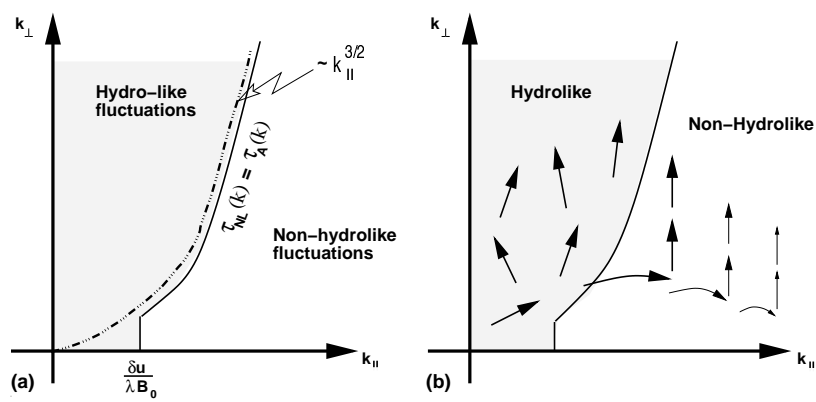

Fig. 2. (a) Partitioning of $\boldsymbol{k}$-space on the basis of whether the nonlinear time at $\boldsymbol{k}$ is less than the (nominal) wave period there: $\tau_{\mathrm{NL}}(\boldsymbol{k}) \lesssim \tau_{\mathrm{A}}(\boldsymbol{k})$. The region where this holds defines the "hydrolike region", wherein nonlinear effects have primacy. Fluctuations in this region are not well described as waves. Conversely, outside the hydrolike region wave effects are of importance. (b) Indication of the strength and direction of spectral transfer at selected points in $\boldsymbol{k}$-space. Within the hydrolike region the transfer is roughly isotropic and analogous to the hydrodynamic situation. Outside this region, parallel transfer is weak, perhaps exponentially so, while perpendicular transfer is still strong due to the resonant transfer mediated by the hydrolike modes (after Oughton et al., 2004).

cally balanced" cascade (Fig. 2a). While this estimate seems to be reasonably accurate for initial conditions and/or forcing restricted to lie within the RMHD region (Maron and Goldreich, 2001; Cho et al., 2002), one may be faced with applications in which the fluctuations are not confined in this way. This leads to an examination of "high-frequency" or non-RMHD couplings (see below).

Building on the critical balance concept, Cho et al. (2002) proposed a specific model for the (axisymmetric) energy spectrum of strong ${ }^{9}$ MHD turbulence:

$E\left(k_{\perp}, k_{\|}\right) \sim k_{\perp}^{-10 / 3} \exp \left(-\frac{\left|k_{\|} L\right|}{\left(k_{\perp} L\right)^{2 / 3}}\right)$,

where $L$ is a characteristic lengthscale for the energycontaining range. This model spectrum is valid for wavevectors above the energy-containing range and has the advantage that it falls off strongly with increasing $\left|k_{\|}\right|$while retaining a strong perpendicular cascade. As stressed by Cho et al. (2002), it is not a unique choice, being postulated rather than derived. Nonetheless, it does provide a good fit to the simulation data they report on.

\subsubsection{Dynamical appearance of quasi-2D turbulence}

A crucial test of whether RMHD ${ }^{10}$ models are indeed central in low-compressibility MHD turbulence is whether an

\footnotetext{
${ }^{9}$ Meaning the turbulent energy is approximately equal to the energy in the mean field.

10 Hereafter we often use RMHD, quasi-2D, and hydrolike as (near) synonyms. However, there are differences in their definitions. For example, RMHD fluctuations are necessarily of small amplitude relative to the mean field, whereas this need not be true for quasi-2D or hydrolike fluctuations. See, for example, Appendix B of Oughton et al. (2004).
}

initially isotropic spectral state will evolve into a state that becomes more like that envisioned in RMHD. For the case that $\boldsymbol{B}_{0}$ is a dc field, this was investigated numerically in incompressible 2D (Shebalin et al., 1983; Grappin, 1986) and later in 3D (Carbone and Veltri, 1990; Oughton et al., 1994; Matthaeus et al., 1996), with consistent results. See also Bondeson (1985). The basic conclusion is that spectral transfer of energy proceeds more rapidly into wavevectors perpendicular to $\boldsymbol{B}_{0}$. Wavevectors parallel to the mean field that are initially unpopulated remain relatively unpopulated because spectral transfer parallel to $\boldsymbol{B}_{0}$ is weak. This can be understood on the basis of resonance arguments, as was first noted by Shebalin et al. (1983). ${ }^{11}$

The basic physics of the Shebalin et al. (1983) picture is correct, and there is evidence that it is valid in 3D as well as driven and slightly compressible low Mach number MHD (Matthaeus et al., 1996, 1998; Galtier et al., 2001). However, Kinney and McWilliams (1998) made the very important observation that the preference for perpendicular spectral transfer extends to modes beyond those that fall into the RMHD category. Put differently, the propagation effect is generally very strong for modes that are not in the RMHD segment of $\boldsymbol{k}$-space, except for those couplings that are resonant in the sense of Shebalin et al. Even high-frequency, wave-like Fourier modes can engage in certain "zero-frequency" couplings, namely those catalyzed by the quasi-2D (or RMHD) modes that form one arm of their resonant triads. Such couplings increase the $k_{\perp}$ of the high-frequency modes, but leave the energy unchanged in the participating RMHD modes (e.g. Kinney and McWilliams, 1998; Matthaeus et al., 1998; Oughton et al., 1998, 2004). See Fig. 2 b.

In order to quantify the weakness of the parallel cascade one can use simulation data to obtain mean wavenumbers computed parallel and perpendicular to an imposed dc magnetic field (assumed parallel to the $z$ axis). We define the mean-square perpendicular wavenumber by

$\left\langle k_{\perp}^{2}\right\rangle_{j}=\frac{\sum_{\boldsymbol{k}} k_{\perp}^{2}\left|\boldsymbol{j}\left(\boldsymbol{k}_{\perp}, k_{z}\right)\right|^{2}}{\sum_{\boldsymbol{k}}\left|\boldsymbol{j}\left(\boldsymbol{k}_{\perp}, k_{z}\right)\right|^{2}}$,

with an analogous definition for its parallel counterpart, $\left\langle k_{z}^{2}\right\rangle_{j}$. Note that a weighting function is used, in this case the electric current density $\boldsymbol{j}(\boldsymbol{k})$, although any other relevant field could have been employed, e.g. $\boldsymbol{v}$ or $\boldsymbol{b}$. Weighting with $\boldsymbol{j}$ emphasizes structure at smaller scales as compared to weighting with $\boldsymbol{b}$. The summations are over all excited wavevectors.

Figure 3 shows the evolution of these ( $\boldsymbol{j}$-weighted) mean wavenumbers, as determined from a set of unforced pseu-

\footnotetext{
11 Nonlinear interactions in incompressible MHD involve triads of wavevectors satisfying $\boldsymbol{k}_{3}=\boldsymbol{k}_{1}+\boldsymbol{k}_{2}$. For strong $\boldsymbol{B}_{0}$, the most effective couplings are those triads that also satisfy a (wave) frequency matching condition. Only oppositely propagating fluctuations interact, so all interacting resonant triads of Fourier modes have at least one member that satisfies $\boldsymbol{k}_{i} \cdot \boldsymbol{B}_{0}=0$ (Shebalin et al., 1983). Necessarily, the other two then have the same $\boldsymbol{k}_{\|}$. Therefore resonant spectral transfer will tend to increase $k_{\perp}$ but not $k_{\|}$.
} 

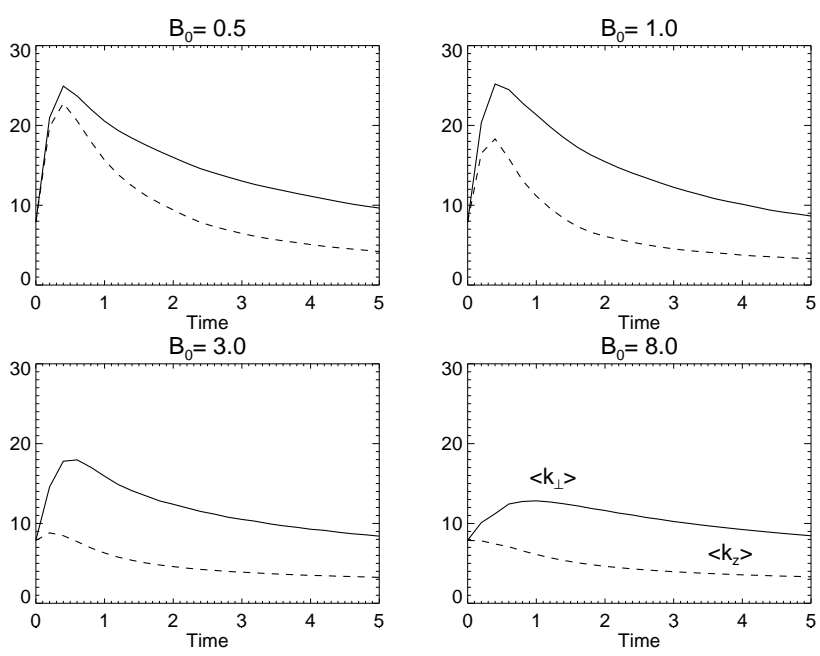

Fig. 3. Evolution of the average parallel and perpendicular wavenumbers for $128^{3}$ incompressible simulations for several values of dc field strength, $B_{0}$. The mean wavenumbers are rms values with a weighting of $|\boldsymbol{j}(\boldsymbol{k})|^{2}$ at each scale; see Eq. (9). Initial conditions for the simulations were identical, with the excited modes band-limited between $|\boldsymbol{k}|=4-20$, having gaussian random phases, and an Alfvén ratio of unity. The $B_{0}=0$ case is not shown, but has the two curves essentially overlain, as is to be expected for isotropy.

dospectral simulations. ${ }^{12}$ Each panel in the figure is for a different value of the dc field strength, where the initial Reynolds numbers $(\approx 400)$ and $\boldsymbol{v}$ and $\boldsymbol{b}$ fields are identical for all runs. The trend towards "freeze-out" of the parallel cascade with increasing $B_{0}$ is clear. Indeed, saturation occurs for $B_{0} \gtrsim 4$ (Shebalin et al., 1983). This is to be contrasted with the behavior of $\sqrt{\left\langle k_{\perp}^{2}\right\rangle}$, which indicates that perpendicular transfer is still strong, although reducing somewhat with increasing $B_{0}$ (perhaps due mostly to the modest Reynolds numbers employed).

Keeping in mind the expectation that physical processes tend to be local, rather than depending on conditions "at infinity," one might ask to what extent these results depend upon the uniformity of the mean magnetic field. One would expect that a strong magnetic field that varied on very large length scales would act, with regard to development of anisotropy, in almost the same way as a uniform dc field. This issue was addressed by Cho and Vishniac (2000) and Milano et al. (2001), who asked whether $\boldsymbol{x}$-space correlation statistics were anisotropic relative to the local magnetic field. Although their approaches were somewhat different, in each case second-order structure functions were used, and the results were consistent with the above picture of the anisotropic development of gradients relative to the mean magnetic field. One can summarize the results as follows. Consider the second-order structure functions

\footnotetext{
12 To make the comparison with $\sqrt{\left\langle k_{z}^{2}\right\rangle}$ fair, Fig. 3 actually plots $\sqrt{\left\langle k_{\perp}^{2}\right\rangle / 2}$, since there are two independent directions in the perpendicular plane.
}

$D_{\|}(r)=\left\langle\left|\boldsymbol{b}\left(\boldsymbol{x}+r \hat{\boldsymbol{e}}_{\|}\right)-\boldsymbol{b}(\boldsymbol{x})\right|^{2}\right\rangle$, where $\hat{\boldsymbol{e}}_{\|}$is a unit vector along the local magnetic field, and $D_{\perp}(r)=\left\langle\left|\boldsymbol{b}\left(\boldsymbol{x}+r \hat{\boldsymbol{e}}_{\perp}\right)-\boldsymbol{b}(\boldsymbol{x})\right|^{2}\right\rangle$ where $\hat{\boldsymbol{e}}_{\perp}$ is a unit vector in any direction perpendicular to the magnetic field. Both studies are consistent with the statement that $D_{\perp}(r)>D_{\|}(r)$. This implies that the variation of the fluctuations perpendicular to the local magnetic field is of smaller scale compared to that along it. One might call this "correlation anisotropy" and it is the $\boldsymbol{x}$-space, local, version of the spectral anisotropy discussed above. Analyses of spectra and/or mean wavenumbers computed relative to the local mean field have also been performed (Maron and Goldreich, 2001; Cho et al., 2002; Muller et al., 2003). For the most part these support the $k_{\perp} \sim k_{\|}^{3 / 2}$ scaling. It is reassuring to find that the physics of the development of anisotropy is, in the end, local.

Studies have also shown that propagation-induced spectral (and correlation) anisotropy is a property of incompressible and nearly incompressible MHD. For example, Matthaeus et al. (1996) found, with strong $B_{0}$, that the solenoidal $(\nabla \cdot v=0)$ part of the velocity field $\boldsymbol{v}$ exhibits spectral anisotropy, while the longitudinal part $(\nabla \cdot v \neq 0)$ remains isotropically distributed in $\boldsymbol{k}$-space. This was confirmed later by Cho and Lazarian (2002), at higher resolution. Evidently this is due to the fact that suppression of spectral transfer is mainly associated with Alfvénic fluctuations, which have the anisotropic dispersion relation $\omega= \pm \boldsymbol{k} \cdot \boldsymbol{B}_{0}$. Interestingly, one can arrive at a complementary result by considering the asymptotic low Mach number limit of nearly incompressible MHD at varying plasma beta (Zank and Matthaeus, 1993). Therein, a regularized asymptotic expansion of the compressible MHD equations is carried out, and the conditions necessary to attain the incompressible limit investigated. A main conclusion, for order one or low plasma beta, is that the limit to incompressibility can occur only if the excited wavevectors become arranged so that $\nabla_{\|} \ll \nabla_{\perp}$ for the solenoidal part of the velocity, and also for the magnetic fluctuations. Departures from this ordering occur at $O\left(M_{S}\right)$, where $M_{s}$ is the turbulent Mach number. This reinforces, from an entirely different perspective, the association of perpendicular spectral (or correlation) anisotropy with the incompressive motions of MHD turbulence.

A consistent conclusion emerges: provided that the turbulence is incompressible or nearly incompressible, MHD turbulence tends to produce gradients perpendicular to a strong magnetic field faster than it produces gradients along the same magnetic field. Generically, this is a consequence of the suppression of parallel spectral transfer by the Alfvén wave propagation effect. Further physical insight is gained from deeper examination of several special cases.

\subsubsection{Weak turbulence}

We mentioned earlier that the high-frequency non-RMHD modes can also engage in resonant nonlinear spectral transfer to higher $k_{\perp}$, a process in which the quasi-2D RMHD modes act as couriers (Fig. 2b; and Fig. 2 in Oughton et al., 2004). 
Now let us change the question to how these highfrequency modes interact when the RMHD modes are nearly absent, i.e. energetically weak. This regime has become known as "weak turbulence" (Galtier et al., 2000, 2002). Nonlinear and resonant couplings are still present, with the three-wave resonances where one mode has $k_{\|}=0$ playing crucial roles. It is noteworthy that perpendicular spectral transfer is still favored, and indeed to leading-order there is no parallel transfer. Standard dimensional analysis methods reveal that the perpendicular spectrum is of the form $k_{\perp}^{-2}$ in the inertial range ( $\mathrm{Ng}$ and Bhattacharjee, 1996, 1997). The same scaling is also obtained using the more rigorous kinetic equation approach (Galtier et al., 2000, 2002).

Interestingly in weak turbulence, $k_{\|}$is more of a parameter than a variable. In particular, to leading-order, the dependence of the spectrum on $k_{\|}$is set by the initial spectrum.

\subsubsection{D turbulence}

Despite numerous simulations at increasingly higher resolution, there is still debate over the value of the inertial range slope of the energy spectrum in incompressible 2D MHD turbulence. Several factors contribute to this debate. Current computing resources are not sufficient to achieve the multidecadal inertial ranges long enough for unambiguous determination of their slope. Differences in numerical resolution, forcing methods (including unforced cases), and models for dissipation (e.g. standard Laplacian diffusion versus hyperdiffusion) may also be causing discrepancies.

In a recent high-resolution $\left(8192^{2}\right)$ study, Biskamp and Schwarz (2001) claimed support for the IK scaling $E(k) \sim k^{-3 / 2}$. However, this has been challenged (Verma et al., 2002; Biskamp, 2002). Further work is clearly called for.

\subsection{Compressible MHD}

There has not been quite as much work in this area, as compared with the incompressible case, although some large numerical studies have appeared recently (e.g. Matthaeus et al., 1996; Vestuto et al., 2003; Lee et al., 2003).

A paper of particular interest is Cho and Lazarian (2002). They performed isothermal simulations, with the plasma beta $\approx 0.2$, and analyzed the data by projecting the fluctuations onto the linear mode polarizations, assuming that this would be statistically valid despite the presence of nonlinear processes. This enabled them to compute structure functions and spectra for each polarization type (conveniently, although perhaps misleadingly, referred to by their linear mode names: Alfvén, slow, and fast modes). They found that spectra for the Alfvén modes were $\sim k^{-5 / 3}$, in agreement with critical balance-type models. Slow mode spectra were also found to have this form, consistent with suggestions that the slow modes should be slaved to the Alfvén modes (Higdon, 1984; Goldreich and Sridhar, 1997; Lithwick and Goldreich, 2001). Fast modes, however, were found to have spectra $\sim k^{-3 / 2}$, a form which they derive using a resonance argument (cf.
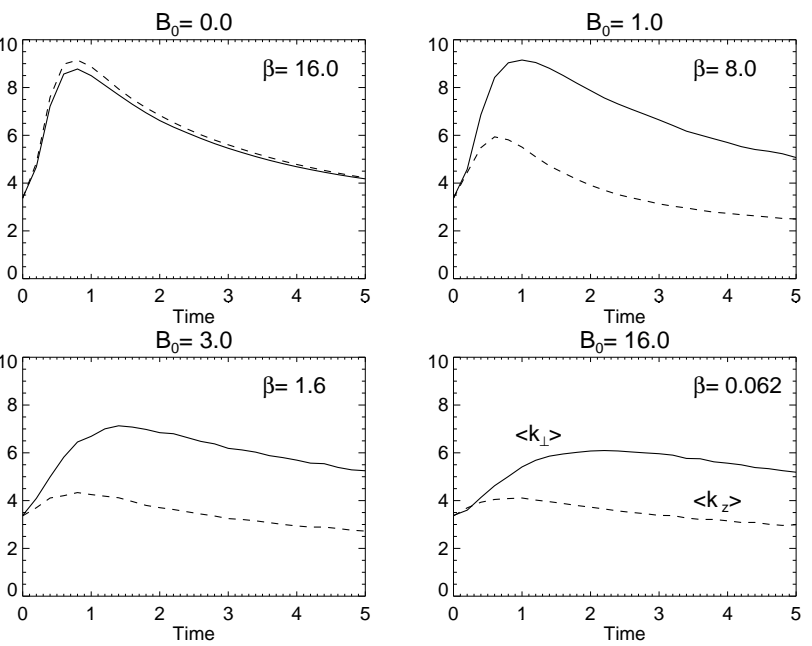

Fig. 4. Evolution of the average parallel and perpendicular wavenumbers for $128^{3} M_{S}=1 / 4$ compressible (polytropic) simulations. The mean wavenumbers are rms values with a weighting of $|\boldsymbol{b}(\boldsymbol{k})|^{2}$ at each scale, which emphasizes large-scale anisotropy. The initial conditions are band-limited between $|\boldsymbol{k}|=4-8$, with gaussian random phases, an Alfvén ratio of 1 , uniform density, and $v$ solenoidal. Initial values for the plasma beta are shown.

Galtier et al., 2001). We note that since, in the low-beta limit, the dispersion relation for fast waves is isotropic, $\omega=k B_{0}$, the classical IK phenomenology can be applied to them, although there are complications since fast modes need not be counter-propagating in order to interact nonlinearly. This is a possible alternative explanation for the $3 / 2$ scaling.

In order to show some of the similarities between incompressible and compressible MHD, as far as parallel and perpendicular cascades are concerned, we have computed mean parallel and perpendicular wavenumbers, defined as in Eq. (9), from a series of compressible (polytropic) simulations (Fig. 4). The behavior depends on system parameters such as the sonic Mach number $\left(M_{s}=u / c_{s}\right)$, the dc field strength $\left(B_{0}\right)$, and the plasma beta $\beta_{\mathrm{p}}=\rho c_{s}^{2} /\left[B_{0}^{2}+\left\langle\boldsymbol{b}^{2}\right\rangle\right]$, defined to include the $\left\langle\boldsymbol{b}^{2}\right\rangle$ contribution to the magnetic pressure. For compressible systems there are also further complicating factors such as how to choose the initial velocity field (solenoidal, longitudinal, or some combination).

Although there are some differences compared to incompressible cases (cf. Fig. 3), the same gross behavior is seen: the parallel cascade weakens as $B_{0}$ is increased (or, as $\beta_{\mathrm{p}}$ decreases). Further investigation of the weakening of the parallel cascade in compressible MHD is underway, but it seems likely that some of the important incompressible results will carry over.

\subsection{Timescales: Forcing and inhomogeneous $\boldsymbol{B}_{0}$ effects}

In this section we summarize some results regarding the impact of various external and/or inhomogeneity related timescales on the development and/or sustainability of turbulent cascades (see also Zhou et al., 2004). 
Table 1. Results from turbulence simulations which support the idea that timescales ordered as in the "Dmitruk inequality", Eq. (10), favor higher levels of turbulent dissipation. Note that the turbulent heating efficiency varies from $0-61 \%$, despite the (average) normalized cross helicity always being in excess of 0.94 . Such high values of $\left\langle\sigma_{c}\right\rangle$ have often been interpreted as evidence for the dominant presence of Alfvén waves, and by inference a relatively unimportant role for turbulence. The results summarized in this table indicate that this is neither a necessary nor a general requirement (after Dmitruk and Matthaeus, 2003).

\begin{tabular}{lccccccc}
\hline Heat. eff. (\%) & 0 & 0 & 2 & 13 & 19 & 46 & 61 \\
$\left\langle\sigma_{c}\right\rangle$ & 1 & 0.94 & 1 & 0.95 & 0.95 & 0.99 & 0.96 \\
\hline$\tau_{\text {refl }} / \tau_{\text {drive }}$ & $\infty$ & 0.1 & 10 & 1 & 1 & 10 & 3.3 \\
$\tau_{\text {cross }} / \tau_{\text {drive }}$ & 1 & 0.1 & 10 & 1 & 1 & 10 & 10 \\
$T_{\text {force }} / \tau_{\text {drive }}$ & 20 & 20 & 2 & 2 & 20 & 20 & 20 \\
\hline
\end{tabular}

Consider a system in which energy is being injected at a boundary with some known energy flux. One can then ask, how efficiently is the energy dissipated by a turbulent cascade? Define this efficiency, $\gamma$, as the rate of energy dissipation by turbulence divided by the injected energy flux.

Using RMHD simulations designed to approximate the situation in a coronal hole, Dmitruk and Matthaeus (2003) have shown that $\gamma$ is subject to constraints between the various timescales characteristic of the system. Specifically, they found that increased values of $\gamma$ are favored when the timescales are suitably ordered:

$\tau_{\mathrm{NL}}<\tau_{\text {drive }}<\tau_{\text {refl }}<\tau_{\text {cross }}<T_{\text {force }}<\tau_{\text {diss }}$.

The geometry of the system is important in defining these timescales: the coronal hole is considered to be forced at its base by photospheric motions, a large-scale vertical magnetic field threads the system, and the Alfvén speed is nonuniform in this direction. The characteristic time for viscous or resistive energy dissipation is $\tau_{\text {diss }}$, while $\tau_{\mathrm{NL}}$ is the usual turbulence nonlinear time. The frequency with which the field lines are shaken at their base determines the period of the forced waves emanating from the boundary, $T_{\text {force }}$. This is quite distinct from the driving timescale, $\tau_{\text {drive }}=\lambda_{0} / u_{0}$, which is associated with the horizontal photospheric motions of typical speed $u_{0}$ and characteristic length $\lambda_{0}$. The reflection timescale, $\tau_{\text {refl }}$, has a reciprocal which is the rate at which upward propagating fluctuations are reflected - due to the gradient in the Alfvén speed - into downward propagating ones, and vice versa. Finally, $\tau_{\text {cross }}$ is the time for a wave to propagate the length of the system. ${ }^{13}$ We refer to Eq. (10) as the Dmitruk inequality.

Table 1 summarizes results from simulations which have timescale orderings which satisfy Eq. (10) to varying degrees. Evidently the better satisfied the Dmitruk inequality is, the more efficiently turbulence dissipates the injected en-

\footnotetext{
13 Note that this is distinct from the period of the wave, unless the wavelength equals the system length.
}

ergy. On the basis of the cross helicity values, all the systems could be classified as Alfvénic - often assumed to mean wavelike. Strikingly, however, the efficacy of the turbulence heating varies from about zero to over $60 \%$.

In work related to these timescale ordering results, and also to the Parker (1972) problem, Dmitruk et al. (2003) have shown that for a stationary forcing pattern at one boundary, the slope of the perpendicular energy spectrum depends strongly on the ratio $m=\tau_{\text {cross }} / \tau_{\text {drive }}$. For $m \ll 1$ the spectral slope is $\approx-3$, while for $m \approx 2$, it is the Kolmogorov value $-5 / 3$. Varying $m$ between these two extremes yields slopes between the above values in an apparently rather continuous fashion. The weak turbulence slope of -2 occurs for $m \approx 1 / 2$.

Note that in a system forced at one boundary, the boundary conditions at the other end of the system can also play an important role in determining whether or not turbulence can be sustained. The key point is that the boundary conditions must allow non-propagating (e.g. 2D) fluctuations to persist in the system, as opposed to propagating through it in relatively short order (Dmitruk et al., 2001).

\section{Observational evidence for a two-component solar wind}

From a turbulence perspective, it is desirable to have access to the full modal three-dimensional wavevector energy spectrum $E^{3 \mathrm{D}}\left(k_{x}, k_{y}, k_{z}\right)$ of the solar wind fluctuations. Unfortunately, as is well-known, this is difficult to achieve using data from a single spacecraft (e.g. Fredricks and Coroniti, 1976). A spacecraft time series can be used to construct a correlation function, and then Fourier transformed to yield the frequency power spectrum $P(f)$. Alternatively, the temporal correlation function can be converted into a spatial one using the Taylor "frozen flow" hypothesis ${ }^{14}$ (Taylor, 1938). The problem, of course, is that this is only a function of one of the three spatial coordinates, namely that parallel to the measurement direction. Fourier transforming this yields the reduced wavenumber power spectrum, ${ }^{15} E^{\text {red }}\left(k_{\text {red }}\right) \equiv \iint E^{3 \mathrm{D}}(\boldsymbol{k}) \mathrm{d} k_{1} \mathrm{~d} k_{2}$, where $k_{\text {red }}=k_{3}$ is along the measurement direction. Except in cases of high symmetry (e.g. isotropic), knowledge of the reduced spectrum is not enough to invert this relationship and determine the more fundamental modal spectrum (e.g. Batchelor, 1970; Fredricks and Coroniti, 1976).

Such information is clearly important since even full vector (amplitude) data may not uniquely determine the geometry of the fluctuations. For example, as noted in Sect. 2.3, minimum variance direction arguments cannot, on their own, be used to distinguish between $2 \mathrm{D}$ and slab fluctuations. A related issue is how to interpret high values of the normalized cross helicity in a system in which turbulence is present (see Sect. 2.5 and Table 1).

Despite these limitations on single-spacecraft datasets, it is sometimes still possible to obtain statistical approximations

\footnotetext{
${ }^{14}$ Valid in the solar wind because of the supersonic flow speed.

${ }^{15}$ Naturally, $P(f)$ and $E^{\text {red }}\left(k_{\text {red }}\right)$ contain the same information.
} 
to $E^{3 \mathrm{D}}(\boldsymbol{k})$. For example, using an ensemble of datasets from $1 \mathrm{AU}$ for which the mean magnetic field was at different angles to the radial (measurement) direction, Matthaeus et al. (1990) computed the autocorrelation function for the magnetic fluctuations $R\left(r_{\|}, r_{\perp}\right)$, assuming axisymmetry about $B_{0}$. A contour plot of this (the so-called "Maltese cross") reveals that there are regions where the contours are approximately parallel to $r_{\|}$and also regions where they are approximately parallel to $r_{\perp}$, supporting the notion that there are at least two distinct types of solar wind fluctuations. These can be usefully idealized as power at approximately $0^{\circ}$ and $90^{\circ}$ to $\boldsymbol{B}_{0}$. Possibilities for the nature of these fluctuations include quasi-2D turbulence $\left(\theta \approx 90^{\circ}\right)$ and quasi-parallel-propagating Alfvén waves $\left(\theta \approx 0^{\circ}\right.$ : the slab component). Clearly this decomposition is not likely to be either exact or complete since there is power at all angles.

Several quantitative studies have also been performed. These include fits of assumed 2D/slab two-component models to cosmic ray mean free path lengths (Bieber et al., 1994), and inertial range energy spectra as a function of the angle between the mean field and the radial (Bieber et al., 1996). Both of these studies found best fits of about $80 \%$ 2D versus $20 \%$ slab, by energy. In addition, nearly incompressible theory (Zank and Matthaeus, 1992b) predicts that the energy in the slab component should scale with the Mach number in a specific way. Using Mach numbers typical of solar wind observations also yields an 80-20 2D/slab partitioning. Collectively, these results indicate that there is abundant, and consistent, evidence for a 2D/slab two-component approximation, with the 2D component energetically dominant. Various two (or more) component models have been employed in efforts to explain the observed evolution of solar wind fluctuations as they are transported outwards by the wind; see, for example, the review article by Tu and Marsch (1995).

In a distinct study, Carbone et al. (1995) analyzed magnetic fluctuations in Alfvénic intervals in terms of the two independent polarizations of $\boldsymbol{b}(\boldsymbol{k})$. Their results also support a two-component perspective, although of a different kind to that of the Maltese cross study, perhaps because of the different data selection policies and assumptions employed in the two studies.

A few simulation studies have also been conducted with a view to understanding how (or if) the two components could develop from particular initial conditions (ICs). For example, Ghosh et al. (1998a,b) found that they could produce Maltese cross-like correlation functions in two different ways. The first involved time-averaging over the evolution associated with ICs consisting of slab waves and (magnetic) pressurebalanced "structures" (these have $\boldsymbol{k} \perp \boldsymbol{B}_{0}$ and $\boldsymbol{b} \| \boldsymbol{B}_{0}$ ). The second way was to have both slab and (magnetic) quasi-2D turbulence present in the ICs. The, perhaps surprising, importance of the initial conditions in this study suggests that it might be possible to draw conclusions about the fluctuations present at the solar wind's "inner boundary" (at or near the Alfvén critical radius), from measurements made well beyond that distance.

Other simulations (Grappin et al., 1993; Grappin and Velli,
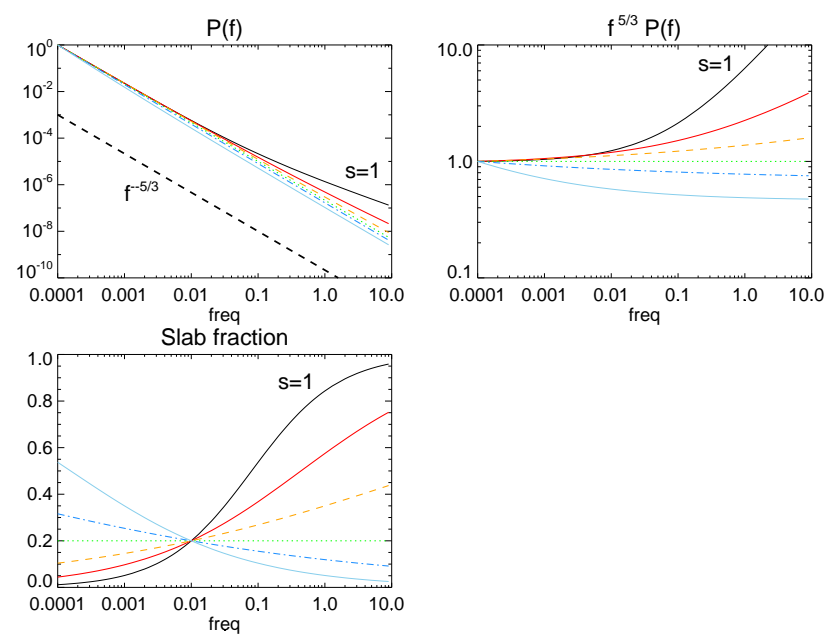

Fig. 5. Power spectra for the $2 \mathrm{D} / \mathrm{slab}$ model, Eq. (11), evaluated with $f_{\text {ref }}=0.01, \psi=45^{\circ}$, and $r_{\mathrm{S}}=0.2$. The different curves are for slab-component slopes of $s=1$ (black), 1.3, 1.5, 5/3, 1.8, and 2.0 (light blue). The slope for the $2 \mathrm{D}$ component is $5 / 3$. Upper panels: Power spectra and compensated power spectra. Lower panel: Fractional contribution of slab component to $P(f)$.

1996; Ruderman et al., 1999; Goldstein et al., 2003; Roberts et al., 1992) have shown that the effects of spherical expansion and/or velocity shear can populate modes with large perpendicular wavenumbers, thus generating or strengthening a perpendicular component in the energy spectrum.

\section{Spectral slopes in the solar wind: Why $5 / 3$ ?}

As noted in the introduction, it is somewhat of a puzzle why a compressible, ionized, collisionless, magnetized plasma like the solar wind is frequently found to have energy spectra with inertial range slopes equal (within error bars) to the incompressible hydrodynamic value of $-5 / 3$. Some theoretical and observational perspectives were considered briefly above. Below we present new results from a simple model which may help explain the commonness of the 5/3 slope.

We model the fluctuations as an admixture of strictly 2D MHD turbulence and strictly parallel-propagating Alfvén waves. The components are assumed to have powerlaw wavenumber spectra with independent slopes and energy levels. Bieber et al. (1996) used fits of such a model to observational data to estimate the slab/2D energy partitioning (cf. Smith (2003)). Here, we investigate, theoretically, how the slope of the (composite) reduced spectrum varies as a function of the field winding angle $\psi$ and the slab/2D partitioning.

Suppose the 2D component is $\sim k^{-q}$, with $q$ fixed at $5 / 3$, while the slab component is $\sim k^{-s}$, with $s$ varied between 1 and 2. Under the above assumptions, the frequency spectrum can then be written (Oughton, 1993; Bieber et al., 1996),

$$
P(f) \propto \frac{\sqrt{2}^{s-q} r_{\mathrm{S}}}{1-r_{\mathrm{S}}}\left(\frac{f_{\mathrm{ref}}}{f}\right)^{s} \cos ^{s-1} \psi+\left(\frac{f_{\mathrm{ref}}}{f}\right)^{q} \sin ^{q-1} \psi .
$$


Table 2. Slopes obtained from least-squares fits to a powerlaw for the two-component model of $P(f)$, with the indicated slab slope and slab fraction at $f_{\text {ref }}$. Fitting is over the range $(0.1-10) \times f_{\text {ref }}$.

\begin{tabular}{cllllll}
\hline slab slope, $s$ & 1 & 1.3 & 1.5 & $5 / 3$ & 1.8 & 2 \\
\hline slab fraction, $r_{\mathrm{S}}$ & \multicolumn{6}{c}{ Best-fit slope } \\
0.05 & 1.62 & 1.65 & 1.66 & 1.67 & 1.67 & 1.68 \\
0.1 & 1.58 & 1.62 & 1.65 & 1.67 & 1.68 & 1.70 \\
0.2 & 1.50 & 1.58 & 1.63 & 1.67 & 1.69 & 1.73 \\
0.3 & 1.43 & 1.55 & 1.62 & 1.67 & 1.71 & 1.76 \\
\hline
\end{tabular}

The constant $r_{\mathrm{S}}$ is the fractional contribution of the slab component to $P\left(f_{\text {ref }}\right)$ at the (arbitrary) reference frequency $f_{\text {ref }}$ and reference angle $\psi=45^{\circ}$. As noted in Sect. 3, previous studies indicate that $r_{\mathrm{S}}=0.05-0.3$ is a useful range to consider for the solar wind. When $s<q$ the $2 \mathrm{D}$ spectrum will dominate for sufficiently low frequencies, and vice versa.

Figure 5 shows spectra from this model for six different values of $s$, all with $r_{\mathrm{S}}=0.2$ and $\psi=45^{\circ}$. From the plots of $P(f)$ and its compensated form $f^{q} P(f)$, it is apparent that provided $|s-q|$ is not too big, there is a range of $f$ near the reference frequency for which the spectrum is $\approx f^{-q}$. Qualitatively similar plots and results are obtained for other values of $r_{\mathrm{S}}$ and $\psi$.

To make a more quantitative comparison, we fit powerlaws to $P(f)$ over the arbitrarily chosen range $0.1 f_{\text {ref }}-10 f_{\text {ref. }}$. The results of these fits, for the same set of $s$ values, $\psi=45^{\circ}$, and four values of $r_{\mathrm{S}}$, are listed in Table 2. It is evident that many of the best-fit slopes are quite close to the 2D slope, despite the fact that the slab fraction can vary significantly over the fitted interval (Fig. 5, lower panel). (Best-fit slopes obtained using larger values of $\psi$ are even closer to the 2D slope, since more of the 2D component is measured in these cases.) This suggests that it could be quite difficult to "reverse engineer" the makeup of the solar wind fluctuations using the observed inertial range slopes.

On the other hand, Bieber et al. (1996) have shown that measuring the power levels as a function of winding angle $\psi$, can provide useful constraints on the energy partitioning of two-component models.

\section{Summary}

We have reviewed classical phenomenologies for MHD turbulence and also more recent developments, with a focus on the importance of (lengthscale) anisotropy with respect to a mean magnetic field. In particular, for typical solar wind parameters, the parallel cascade is likely to be rather weak, limiting the effectiveness of heating mechanisms which rely on power being cascaded to the small parallel lengthscales associated with high-frequency Alfvén and ion-cyclotron waves.

Also reviewed were results regarding the impact of boundary conditions and timescales associated with forcing and/or inhomogeneous mean fields on inertial range slopes. Sum- marizing, we note that a wide range of spectral slopes can occur, depending on the contributions of the above factors. Related to this is the efficiency of turbulence at dissipating injected energy, which is also rather wide ranging, for similar reasons.

The observational support for two-component, particularly "slab plus 2D", descriptions of solar wind fluctuations was summarized. Using a simple two-component model we demonstrated that it can be relatively easy to get inertial range slopes close to the typically observed value of $5 / 3$, when the actual spectrum consists of the sum of a $5 / 3$ piece and a second, distinct, powerlaw contribution. How easy this is depends on parameters such as the relative contributions of the two components over a given frequency range. Together with other results reviewed herein, this indicates that care should be exercised when using the observed slopes to draw conclusions about the nature, type, or number of fluctuation components present in the solar wind.

Unfortunately, space constraints have precluded a review of work related to important topics such as parametric decay (e.g. Malara et al., 2001), and the expanding box model (Grappin et al., 1993; Grappin and Velli, 1996).

Also not discussed in any detail was the important issue of the observed heating of solar wind fluctuations to well above adiabatic levels (e.g. Richardson and Smith, 2003; Smith et al., 2001). The role that cascades, both parallel and perpendicular, play in this heating remains to be fully determined and is the object of much current work (e.g. Tu and Marsch, 1997; Leamon et al., 2000).

Acknowledgements. This work was been supported by grants from the NZ Marsden Fund (02-UOW-050 MIS), NASA (NNG04GA54G), and the NSF (ATM-0105254).

Edited by: J. Büchner

Reviewed by: M. Goldstein

\section{References}

Batchelor, G. K.: The Theory of Homogeneous Turbulence, CUP, Cambridge, 1970.

Belcher, J. W. and Davis Jr., L.: Large-amplitude Alfvén Waves in the Interplanetary Medium, 2, J. Geophys. Res., 76, 3534-3563, 1971.

Bieber, J. W., Matthaeus, W. H., Smith, C. W., Wanner, W., Kallenrode, M., and Wibberenz, G.: Proton and Electron Mean Free Paths: The Palmer consensus revisited, Astrophys. J., 420, 294 306, 1994.

Bieber, J. W., Wanner, W., and Matthaeus, W. H.: Dominant TwoDimensional Solar Wind Turbulence with implications for cosmic ray transport, J. Geophys. Res., 101, 2511-2522, 1996.

Biskamp, D.: Response to "Comment on 'On two-dimensional magnetohydrodynamic turbulence", Phys. Plasmas, 9, 14861487, 2002.

Biskamp, D.: Magnetohydrodynamic Turbulence, CUP, 2003.

Biskamp, D. and Schwarz, E.: On two-dimensional magnetohydrodynamic turbulence, Phys. Plasmas, 8, 3282-3292, 2001.

Bondeson, A.: Cascade properties of shear Alfvén turbulence, Phys. Fluids, 28, 2406-2411, 1985. 
Carbone, V. and Veltri, P.: A shell model for anisotropic Magnetohydrodynamic Turbulence, Geophys. \& Astrophys. Fluid Dynam., 52, 153-181, 1990.

Carbone, V., Malara, F., and Veltri, P.: A model for the threedimensional magnetic field correlation spectra of low-frequency solar wind fluctuations during Alfvénic periods, J. Geophys. Res., 100, 1763-1778, 1995.

Cho, J. and Lazarian, A.: Compressible sub-Alfvénic MHD Turbulence in low- $\beta$ plasmas, Phys. Rev. Lett., 88, 245 001, 2002.

Cho, J. and Vishniac, E. T.: The anisotropy of magnetohydrodynamic Alfvénic turbulence, Astrophys. J., 539, 273-282, 2000.

Cho, J., Lazarian, A., and Vishniac, E. T.: Simulations of magnetohydrodynamic Turbulence in a strongly magnetized region, Astrophys. J., 564, 291-301, 2002.

Coleman, P. J.: Turbulence, Viscosity, and Dissipation in the Solar Wind Plasma, Astrophys. J., 153, 371-388, 1968.

Dmitruk, P. and Matthaeus, W. H.: Low-Frequency Waves and Turbulence in an Open Magnetic Region: Timescales and Heating Efficiency, Astrophys. J., 597, 1097-1105, 2003.

Dmitruk, P., Matthaeus, W. H., Milano, L. J., and Oughton, S.: Conditions for sustainment of magnetohydrodynamic turbulence driven by Alfvén waves, Phys. Plasmas, 8, 2377-2384, 2001.

Dmitruk, P., Gómez, D. O., and Matthaeus, W. H.: Energy spectrum of turbulent fluctuations in boundary driven reduced magnetohydrodynamics, Phys. Plasmas, 10, 3584-3591, 2003.

Dobrowolny, M., Mangeney, A., and Veltri, P.: Fully Developed Anisotropic Hydromagnetic Turbulence in Interplanetary Space, Phys. Rev. Lett., 45, 144-147, 1980.

Fredricks, R. W. and Coroniti, F. V.: Ambiguities in the deduction of rest frame fluctuation spectrums from spectrums computed in moving frames, J. Geophys. Res., 81, 5591-5595, 1976.

Frisch, U.: Turbulence, CUP, Cambridge, 1995

Galtier, S., Nazarenko, S. V., Newell, A. C., and Pouquet, A.: A weak turbulence theory for incompressible magnetohydrodynamics, J. Plasma Phys., 63, 447-488, 2000.

Galtier, S., Nazarenko, S. V., and Newell, A. C.: On wave turbulence in MHD, Nonlin. Process. Geophys., 8, 141-150, 2001.

Galtier, S., Nazarenko, S. V., Newell, A. C., and Pouquet, A.: Anisotropic Turbulence of Shear-Alfvén Waves, Astrophys. J., 564, L49-L52, 2002.

Ghosh, S., Matthaeus, W. H., Roberts, D. A., and Goldstein, M. L.: The evolution of slab fluctuations in the presence of pressure balanced structures and velocity shears, J. Geophys. Res., 103, 23 691-23 704, 1998a.

Ghosh, S., Matthaeus, W. H., Roberts, D. A., and Goldstein, M. L.: Waves, structures, and the appearance of two-component turbulence in the solar wind, J. Geophys. Res., 103, 23 705-23 715, $1998 \mathrm{~b}$

Goldreich, P. and Sridhar, S.: Toward a theory of interstellar turbulence: II. Strong Alfvénic Turbulence, Astrophys. J., 438, 763$775,1995$.

Goldreich, P. and Sridhar, S.: Magnetohydrodynamic turbulence revisited, Astrophys. J., 485, 680-688, 1997.

Goldstein, M. L., Roberts, D. A., and Matthaeus, W. H.: Magnetohydrodynamic turbulence in the solar wind, Ann. Rev. Astron. Astrophys., 33, 283-325, 1995.

Goldstein, M. L., Roberts, D. A., and Deane, A.: The effect of microstreams on Alfvénic fluctuations in the solar wind, in Solar Wind Ten, edited by M. Velli, R. Bruno, and F. Malara, vol. 679, pp. 405-408, AIP, Melville, NY, 2003.

Grappin, R.: Onset and decay of two-dimensional magnetohydrodynamic turbulence with velocity-magnetic field correlation,
Phys. Fluids, 29, 2433-2443, 1986.

Grappin, R. and Velli, M.: Waves and Streams in the Expanding Solar Wind, J. Geophys. Res., 101, 425-444, 1996.

Grappin, R., Frisch, U., Léorat, J., and Pouquet, A.: Alfvénic Fluctuations as Asymptotic States of MHD Turbulence, Astron. Astrophys., 105, 6-14, 1982.

Grappin, R., Pouquet, A., and Léorat, J.: Dependence of MHD Turbulence Spectra on the Velocity field-Magnetic field Correlation, Astron. Astrophys., 126, 51-58, 1983.

Grappin, R., Velli, M., and Mangeney, A.: Nonlinear Wave Evolution in the Expanding Solar Wind, Phys. Rev. Lett., 70, 2190 2193, 1993.

Higdon, J. C.: Density Fluctuations in the interstellar medium: Evidence for anisotropic magnetogasdynamic turbulence. I Model and astrophysical sites, Astrophys. J., 285, 109-123, 1984

Horbury, T., Balogh, A., Forsyth, R. J., and Smith, E. J.: Anisotropy of inertial range turbulence in the polar heliosphere, Geophys. Rev. Lett., 22, 3405-3408, 1995.

Hossain, M., Gray, P. C., Pontius Jr., D. H., Matthaeus, W. H., and Oughton, S.: Phenomenology for the Decay of EnergyContaining Eddies in Homogeneous MHD Turbulence, Phys. Fluids, 7, 2886-2904, 1995.

Iroshnikov, P. S.: Turbulence of a conducting fluid in a strong magnetic field, Soviet Astron., 7, 566-571, 1964.

Jokipii, J. R.: Cosmic-ray propagation. I. Charged particles in a random magnetic field, Astrophys. J., 146, 480-487, 1966.

Kadomtsev, B. B. and Pogutse, O. P.: Nonlinear helical perturbations of a plasma in the tokamak, Sov. Phys.-JETP, 38, 283-290, 1974.

Kinney, R. and McWilliams, J. C.: Turbulent cascades in anisotropic magnetohydrodynamics, Phys. Rev. E, 57, 71117121, 1998.

Klein, L. W., Roberts, D. A., and Goldstein, M. L.: Anisotropy and Minimum Variance Directions of Solar Wind Fluctuations in the Outer Heliosphere, J. Geophys. Res., 96, 3779-3788, 1991.

Kolmogorov, A. N.: Local Structure of Turbulence in an Incompressible Viscous Fluid at Very High Reynolds Numbers, Dokl. Akad. Nauk SSSR, 30, 301-305, [Reprinted in Proc. R. Soc. London, Ser. A 434, 9-13 (1991)], 1941.

Kraichnan, R. H.: Inertial-Range Spectrum of Hydromagnetic Turbulence, Phys. Fluids, 8, 1385-1387, 1965.

Leamon, R. J., Matthaeus, W. H., Smith, C. W., Zank, G. P., Mullan, D. J., and Oughton, S.: MHD Driven Kinetic Dissipation in the Solar Wind and Corona, Astrophys. J., 537, 1054-1062, 2000.

Lee, H., Ryu, D., Kim, J., Jones, T. W., and Balsara, D.: Numerical Study of Compressible Magnetohydrodynamic Turbulence in Two Dimensions, Astrophys. J., 594, 627-636, 2003.

Lesieur, M.: Turbulence in Fluids, Kluwer, Dordrecht, The Netherlands, 2nd edn., 1990.

Lithwick, Y. and Goldreich, P.: Compressible Magnetohydrodynamic Turbulence in Interstellar Plasmas, Astrophys. J., 562, 279-296, 2001.

Malara, F., Primavera, L., and Veltri, P.: Nonlinear evolution of the parametric instability: Numerical predictions versus observations in the heliosphere, Nonlin. Proc. Geophys., 8, 159-166, 2001 ,

\section{SRef-ID: 1607-7946/npg/2001-8-159}

Maron, J. and Goldreich, P.: Simulations of incompressible magnetohydrodynamic turbulence, Astrophys. J., 554, 1175-1196, 2001

Matthaeus, W. H. and Zhou, Y.: Extended Inertial Range Phenomenology of Magnetohydrodynamic Turbulence, Phys. Fluids 
B, 1, 1929-1931, 1989.

Matthaeus, W. H., Goldstein, M. L., and Roberts, D. A.: Evidence for the Presence of Quasi-Two-Dimensional Nearly Incompressible fluctuations in the Solar Wind, J. Geophys. Res., 95, $20673-$ $20683,1990$.

Matthaeus, W. H., Oughton, S., Pontius, D., and Zhou, Y.: Evolution of Energy Containing Turbulent Eddies in the solar wind, J. Geophys. Res., 99, 19267-19287, 1994.

Matthaeus, W. H., Bieber, J. W., and Zank, G. P.: Unquiet on any front: Anisotropic turbulence in the solar wind, Rev. Geophys. Supp., 33, 609-614, 1995.

Matthaeus, W. H., Ghosh, S., Oughton, S., and Roberts, D. A.: Anisotropic Three-Dimensional MHD Turbulence, J. Geophys. Res., 101, 7619-7629, 1996.

Matthaeus, W. H., Oughton, S., Ghosh, S., and Hossain, M.: Scaling of Anisotropy in Hydromagnetic Turbulence, Phys. Rev. Lett., 81, 2056-2059, 1998.

Milano, L. J., Matthaeus, W. H., Dmitruk, P., and Montgomery, D. C.: Local anisotropy in incompressible magnetohydrodynamic turbulence, Phys. Plasmas, 8, 2673-2681, 2001.

Montgomery, D. C.: Major disruption, inverse cascades, and the Strauss equations, Physica Scripta, T2/1, 83-88, 1982.

Montgomery, D. C. and Turner, L.: Anisotropic magnetohydrodynamic turbulence in a strong external magnetic field, Phys. Fluids, 24, 825-831, 1981.

Muller, W.-C., Biskamp, D., and Grappin, R.: Statistical anisotropy of magnetohydrodynamic turbulence, Phys. Rev. E, 67, 066302 , 2003.

$\mathrm{Ng}, \mathrm{C}$. S. and Bhattacharjee, A.: Interaction of Shear-Alfvén wave packets: Implications for weak magnetohydrodynamic turbulence in astrophysical plasmas, Astrophys. J., 465, 845-854, 1996.

Ng, C. S. and Bhattacharjee, A.: Scaling of anisotropic spectra due to the weak interaction of Shear-Alfvén wave packets, Phys. Plasmas, 4, 605-610, 1997.

Obukhov, A. M.: On the energy distribution in the spectrum of a turbulent flow, Dokl. Akad. Nauk SSSR, 32, 22-24, [C.R. (Dokl.) Acad. Sci. URSS 32, 19 (1963)], 1941.

Oughton, S.: Transport of Solar Wind Fluctuations: A Turbulence Approach, Ph.D. thesis, University of Delaware, Newark, Delaware, 19716, 1993.

Oughton, S., Priest, E. R., and Matthaeus, W. H.: The Influence of a mean magnetic field on three-dimensional MHD turbulence, $\mathrm{J}$. Fluid Mech., 280, 95-117, 1994.

Oughton, S., Ghosh, S., and Matthaeus, W. H.: Scaling of Spectral Anisotropy with Magnetic Field Strength in Decaying MHD Turbulence, Phys. Plasmas, 5, 4235-4242, 1998.

Oughton, S., Dmitruk, P., and Matthaeus, W. H.: Reduced Magnetohydrodynamics and parallel spectral transfer, Phys. Plasmas, 11, 2214-2225, 2004.

Parker, E. N.: Dynamics of the Interplanetary Gas and Magnetic Fields, Astrophys. J., 128, 664-676, 1958.

Parker, E. N.: Topological Disspation and the Small-Scale Fields in Turbulent Gases, Astrophys. J., 174, 499-510, 1972.

Pouquet, A., Frisch, U., and Léorat, J.: Strong MHD Helical Turbulence and the Nonlinear Dynamo Effect, J. Fluid Mech., 77, 321-354, 1976.

Pouquet, A., Meneguzzi, M., and Frisch, U.: Growth of Correlations in Magnetohydrodynamic Turbulence, Phys. Rev. A, 33, 4266-4275, 1986.
Richardson, J. D. and Smith, C. W.: The radial temperature profile of the solar wind, Geophys. Rev. Lett., 30, 1206, doi:10.1029/2002GL016 551, 2003.

Roberts, D. A., Goldstein, M. L., Matthaeus, W. H., and Ghosh, S.: Velocity Shear Generation of Solar Wind Turbulence, J. Geophys. Res., 97, 17 115-17 130, 1992.

Robinson, D. and Rusbridge, M.: Structure of turbulence in the Zeta plasma, Phys. Fluids, 14, 2499-2511, 1971.

Ruderman, M. S., Goldstein, M. L., Roberts, D. A., Deane, A., and Ofman, L.: Alfvén wave phase mixing driven by velocity shear in two-dimensional open magnetic configurations, J. Geophys. Res., 104, 17 057-17 068, 1999.

Shebalin, J. V., Matthaeus, W. H., and Montgomery, D.: Anisotropy in MHD Turbulence due to a Mean Magnetic Field, J. Plasma Phys., 29, 525-547, 1983.

Smith, C. W.: The Geometry of Turbulent Magnetic Fluctuations at High Heliographic Latitude, in Solar Wind Ten, edited by M. Velli, R. Bruno, and F. Malara, vol. 679, pp. 413-416, AIP, Melville, NY, 2003.

Smith, C. W., Matthaeus, W. H., Zank, G. P., Ness, N. F., Oughton, S., and Richardson, J. D.: Heating of the Low-Latitude Solar Wind by Dissipation of Turbulent Magnetic Fluctuations, J. Geophys. Res., 106, 8253-8272, 2001.

Strauss, H. R.: Nonlinear, three-dimensional magnetohydrodynamics of noncircular tokamaks, Phys. Fluids, 19, 134-140, 1976.

Taylor, G. I.: The spectrum of turbulence, Proc. Roy. Soc. Lond. A, 164, 476-490, 1938.

Tu, C.-Y. and Marsch, E.: MHD Structures, Waves and Turbulence in the Solar Wind, Space Sci. Rev., 73, 1-210, 1995.

Tu, C.-Y. and Marsch, E.: Two-Fluid Model for heating of the solar corona and acceleration of the solar wind by high-frequency Alfvén waves, Solar Phys., 171, 363-391, 1997.

Tu, C.-Y., Pu, Z.-Y., and Wei, F.-S.: The Power Spectrum of Interplanetary Alfvénic Fluctuations: Derivation of the Governing Equation and its Solution, J. Geophys. Res., 89, 9695-9702, 1984.

Velli, M., Bruno, R., and Malara, F., eds.: Solar Wind Ten, vol. 679, AIP, Melville, NY, 2003.

Veltri, P. and Malara, F.: Theoretical models for MHD turbulence in the solar wind, Il Nuovo Cimento, 20, 859-869, 1997.

Verma, M. K., Dar, G., and Eswaran, V.: Comment on "On twodimensional magnetohydrodynamic turbulence", Phys. Plasmas, 9, 1484-1485, 2002.

Vestuto, J. G., Ostriker, E. C., and Stone, J. M.: Spectral Properties of Compressible Magnetohydrodynamic Turbulence from Numerical Simulations, Astrophys. J., 590, 858-873, 2003.

Zank, G. P. and Matthaeus, W. H.: The Equations of Reduced Magnetohydrodynamics, J. Plasma Phys., 48, 85-100, 1992a.

Zank, G. P. and Matthaeus, W. H.: Waves and Turbulence in the Solar Wind, J. Geophys. Res., 97, 17 189-17 194, 1992 b.

Zank, G. P. and Matthaeus, W. H.: Nearly Incompressible Fluids. II: Magnetohydrodynamics, Turbulence, and Waves, Phys. Fluids A, 5, 257-273, 1993.

Zhou, Y., Matthaeus, W. H., and Dmitruk, P.: Magnetohydrodynamic turbulence and time scales in astrophysical and space plasmas, Rev. Mod. Phys., 76, 1015-1035, 2004.

Zweben, S., Menyuk, C., and Taylor, R.: Small-scale magnetic fluctuations inside the Macrotor tokamak, Phys. Rev. Lett., 42, 1270 1274, 1979. 